\title{
Generalized Alamouti Codes for Trading Quality of Service against Data Rate in MIMO UMTS
}

\author{
Christoph F. Mecklenbräuker \\ Forschungszentrum Telekommunikation Wien (ftw), Donau-City Straße 1, 1220 Vienna, Austria \\ Email: cfm@ftw.at \\ Markus Rupp \\ Institut für Nachrichtentechnik und Hochfrequenztechnik, Technische Universität Wien, \\ Gusshausstraße 25-29, 1040 Vienna, Austria \\ Email:mrupp@nt.tuwien.ac.at
}

Received 17 December 2002; Revised 26 August 2003

\begin{abstract}
New space-time block coding schemes for multiple transmit and receive antennas are proposed. First, the well-known Alamouti scheme is extended to $N_{T}=2^{m}$ transmit antennas achieving high transmit diversity. Many receiver details are worked out for four and eight transmit antennas. Further, solutions for arbitrary, even numbers $\left(N_{T}=2 k\right)$ of transmit antennas are presented achieving decoding advantages due to orthogonalization properties while preserving high diversity. In a final step, such extended Alamouti and BLAST schemes are combined, offering a continuous trade-off between quality of service (QoS) and data rate. Due to the simplicity of the coding schemes, they are very well suited to operate under UMTS with only very moderate modifications in the existing standard. The number of supported antennas at transmitter alone is a sufficient knowledge to select the most appropriate scheme. While the proposed schemes are motivated by utilization in UMTS, they are not restricted to this standard.
\end{abstract}

Keywords and phrases: mobile communications, space-time block codes, spatial multiplexing.

\section{INTRODUCTION}

One of the salient features of UMTS is the provisioning of moderately high data rates for packet switched data services. In order to maximize the number of satisfied users, an efficient resource assignment to the subscribers is desired allowing flexible sharing of the radio resources. Such schemes must address the extreme variations of the link quality. Standardization of UMTS is progressing steadily, and various schemes for transmit diversity [1] and high-speed downlink packet access (HSDPA) with multiple transmit and receive antennas (MIMO) schemes [2] are currently under debate within the Third Generation Partnership Project (http://www.3gpp.org/).

Recently, much attention has been paid to wireless MIMO systems, (cf. $[3,4,5]$ ). In $[6,7]$, it was shown that the wireless MIMO channel potentially has a much higher capacity than was anticipated previously. In $[8,9,10]$, spacetime coding (STC) schemes were proposed that efficiently utilize such channels. Alamouti [11] introduced a very simple scheme allowing transmissions from two antennas with the same data rate as on a single antenna but increasing the diversity at the receiver from one to two in a flat-fading channel. While the scheme works for BPSK even with four and eight antennas, it was proven that for QPSK, only the two-transmit-antenna scheme offers the full diversity gain $[8,12]$.

In order to evaluate the (single-) symbol error probability for a random channel $\mathbf{H}$ with $N_{T}$ statistically independent transmission paths with zero-mean channel coefficients $h_{k}$ $\left(k=1, \ldots, N_{T}\right)$ of equal variance, ${ }^{1}$ known results from literature for maximum likelihood (ML) decoding of uncoded QPSK (with gray-code labelling) can be employed [13]:

$$
\begin{aligned}
\operatorname{BER}_{\mathrm{ML}} & =\frac{1}{2} \mathrm{E}_{\mathrm{H}}\left[\operatorname{erfc}\left(\sqrt{\frac{E_{b}}{2 N_{0}} \sum_{k=1}^{N_{T}}\left|h_{k}\right|^{2}}\right)\right] \\
& =\frac{1}{2} \mathrm{E}_{\alpha_{\mathrm{ML}}}\left[\operatorname{erfc}\left(\sqrt{\frac{\alpha_{\mathrm{ML}}}{\sigma_{V}^{2}}}\right)\right] .
\end{aligned}
$$

Here the fading factor $\alpha_{\mathrm{ML}}$ is introduced as a random variable with $\chi_{2 N_{T}}^{2}$ density, the index indicating $2 N_{T}$ degrees of freedom, that is, a diversity order of $N_{T}$. In case of independent complex Gaussian distributed variables $h_{k}$, the follow-

\footnotetext{
${ }^{1}$ We normalize $\sum_{k=1}^{N_{T}} \mathrm{E}\left[\left|h_{k}\right|^{2}\right]=1$.
} 
ing explicit result for QPSK modulation is obtained according to $[13$, Section 14.4 , equations (15)]:

$$
\begin{aligned}
\mathrm{BER}_{\mathrm{ML}} & =\frac{1}{2} \int_{0}^{\infty} \operatorname{erfc}\left(\sqrt{x \frac{E_{b}}{2 N_{0}}}\right) \frac{x^{N_{T}-1}}{\Gamma\left(N_{T}\right)} e^{-x} d x \\
& =\left[\frac{1-\mu}{2}\right]^{N_{T}} \sum_{k=0}^{N_{T}-1}\left(\begin{array}{c}
N_{T}-1+k \\
k
\end{array}\right)\left(\frac{1+\mu}{2}\right)^{k}, \\
\mu & =\sqrt{\frac{E_{b} / N_{0}}{N_{T}+E_{b} / N_{0}}} .
\end{aligned}
$$

In contrast to this behavior, the performance for a linear zeroforeing $(\mathrm{ZF})$ receiver is different. The bit error rate (BER) for a ZF receiver with $N_{T}$ transmit and $N_{R}$ receive antennas is given by [14]

$$
\mathrm{BER}_{\mathrm{ZF}}=\frac{1}{2} \mathrm{E}_{\alpha_{\mathrm{ZF}}}\left[\operatorname{erfc}\left(\sqrt{\frac{\alpha_{\mathrm{ZF}}}{\sigma_{V}^{2}}}\right)\right],
$$

with $\alpha_{\mathrm{ZF}}$ being $\chi^{2}$-distributed with $2\left(N_{T}-N_{R}+1\right)$ degrees of freedom rather than $2 N_{T}$. A good overview of the various single symbol error performances is given in [15] and some early results on multiple symbol errors in [16]. The proposed coding schemes of this paper will be compared with these results for uncoded transmissions. In particular, selecting space-time codes will result in different degrees of freedom for the resulting fading factor $\alpha$ when compared to (1) and (4).

The paper is composed as follows. In Section 2, the wellknown Alamouti scheme is introduced setting the notation for the remaining of the paper. In Section 3, the Alamouti space-time codes for transmission diversity is extended recursively to $M=2^{m}$ antenna elements at the transmitter. While it is well known that the resulting transmission matrix for flat-fading looses its orthogonality for $m \geq 2$, it is shown that the loss in orthogonality for the new schemes is not severe when utilizing gray-coded QPSK modulation. Starting with a four-antenna scheme in Section 3, it will be demonstrated that linear receivers perform close to the theoretical bound for four-path diversity offering significant gain over the two-antenna case proposed by Alamouti. Even more interestingly, linear interference suppression can be implemented at low-complexity because the channel matrix exhibits a high degree of structure, enabling factorization in closed-form. In Section 4, this observation is generalized to extended Alamouti schemes for an arbitrary number of transmit antennas $N_{T}=2^{m}$ preserving as much orthogonality as possible. In particular, results will be presented for the case $N_{T}=8$. Transmission schemes with more than one receive antenna will be considered in Section 5 and it will be shown that even in cases with $N_{T} \neq 2^{m}$ transmit antennas, preservation of orthogonality is possible. Variable bit rate services and bursty packet arrivals are handled flexibly in UMTS by dynamically changing the spreading factor in conjunction with the transmit power, thus preserving an average $E_{b} / N_{0}$, but without changing the diversity order and outage probability. A combination of BLAST and extended Alamouti schemes is proposed in Section 6 that makes use of the existing diversity in a flexible manner, trading diversity gain against data rate and thus augmenting the diversity order and outage probability for fulfilling the quality of service (QoS) requirements. Not considered in this paper is the impact of the modulation scheme on the achieved diversity. It is well known that a certain rank criterion [8] needs to be satisfied in order to utilize full channel diversity in MIMO systems.

\section{ALAMOUTI SCHEME}

A very simple but effective scheme for two $\left(N_{T}=2\right)$ antennas achieving a diversity gain of two was introduced by Alamouti $[8,11]$. It works by sending the sequence $\left\{s_{1}, s_{2}^{*}\right\}$ on the first antenna and $\left\{s_{2},-s_{1}^{*}\right\}$ on the other. Assuming a flat-fading channel and denoting the two channel coefficients by $h_{1}$ and $h_{2}$, the received vector $\mathbf{r}$ is formed by stacking two consecutive data samples $\left[r_{1}, r_{2}\right]^{T}$ in time:

$$
\mathbf{r}=\mathbf{S h}+\overline{\mathbf{v}}
$$

Here, the symbol block $\mathbf{S}$ and the channel vector $\mathbf{h}$ are defined as follows:

$$
\mathbf{S}=\left[\begin{array}{cc}
s_{1} & s_{2} \\
s_{2}^{*} & -s_{1}^{*}
\end{array}\right], \quad \mathbf{h}=\left[\begin{array}{l}
h_{1} \\
h_{2}
\end{array}\right] .
$$

This can be reformulated as

$$
\left[\begin{array}{l}
r_{1} \\
r_{2}^{*}
\end{array}\right]=\left[\begin{array}{cc}
h_{1} & h_{2} \\
-h_{2}^{*} & h_{1}^{*}
\end{array}\right]\left[\begin{array}{l}
s_{1} \\
s_{2}
\end{array}\right]+\left[\begin{array}{c}
v_{1} \\
v_{2}^{*}
\end{array}\right]
$$

or in short notation:

$$
\mathrm{y}=\mathrm{Hs}+\mathbf{v}
$$

where the vector $\mathbf{y}=\left[r_{1}, r_{2}^{*}\right]^{T}$ is introduced. The resulting channel matrix $\mathbf{H}$ is orthogonal, that is, $\mathbf{H}^{H} \mathbf{H}=\mathbf{H} \mathbf{H}^{H}=$ $h^{2} \mathbf{I}_{2}$, where the $2 \times 2$ identity matrix $\mathbf{I}_{2}$ as well as the gain of the channel $h^{2}=\left|h_{1}\right|^{2}+\left|h_{2}\right|^{2}$ are introduced. The transmitted symbols can be computed by the $\mathrm{ZF}$ approach

$$
\hat{\mathbf{s}}=\left[\mathbf{H}^{H} \mathbf{H}\right]^{-1} \mathbf{H}^{H} \mathbf{y}=\frac{1}{h^{2}} \mathbf{H}^{H} \mathbf{y}=\mathbf{s}+\left[\mathbf{H}^{H} \mathbf{H}\right]^{-1} \mathbf{H}^{H} \mathbf{v},
$$

revealing a noise filtering. Note that due to the particular structure of $\mathbf{H}$, the two noise components are orthogonal. For a fixed channel matrix $\mathbf{H}$ and complex-valued Gaussian noise $\mathbf{v}$, it can be concluded that they are both i.i.d. and thus are two decoupled noise components. The noise variance for each of the two symbols is given by $2 \sigma_{V}^{2} / h^{2}$. Comparing to the optimal ML result for two-path diversity, the results are identical indicating that with a simple ZF receiver technique, the full two-path diversity of the transmission system can be obtained. Using complex-valued modulation, only for the two-antenna scheme such an improvement is possible. Only in the case of binary transmission, higher schemes with four and eight antennas exist [12]. In UMTS, QPSK is utilized on CDMA preventing perfectly orthogonal schemes with an improvement larger than a diversity of two. 


\section{FOUR-ANTENNA SCHEME}

In UMTS with frequencies around $2 \mathrm{GHz}$, four or even eight antennas are quite possible at the base stations and two or four antennas at the mobile [17]. Since the number of antennas will vary among base stations and mobile devices, it is vital to design a flexible MIMO transmission scheme supporting various multielement antennas. As a minimum requirement, the mobile station might only be informed about the number of transmit antennas at the base station. Based on its own number of receive antennas, it can then decide which decoding algorithm to apply. Some codes offer complexity proportional to the number of receive antennas, for example, cyclic spacetime codes [18]. Another example being Hadamard codes, retransmitting the symbols in a specific manner. For the case of four transmit antennas, the resulting matrix becomes $\left[s_{1}, s_{2}, s_{3}, s_{4} ; s_{1}, s_{2},-s_{3},-s_{4} ; s_{1},-s_{2},-s_{3}, s_{4} ;-s_{1}, s_{2},-s_{3}, s_{4}\right]$. In such schemes, the receiver can be built with very lowcomplexity, and higher diversity is achievable with more receiver antennas. However, by only utilizing multiple receiver antennas, the maximum possible diversity is not utilized in such systems unless transmit diversity is utilized as well.

In the following, simple block codes supporting much higher diversity in a four transmit antenna scheme for UMTS are proposed which do take advantage of additional transmit diversity. $^{2}$

Proposition 1. Starting with the $2 \times 2$-Alamouti scheme, the following recursive construction rule (similar to the construction of a complex Walsh-Hadamard code) is applied:

$$
\left[\begin{array}{cc}
h_{1} & h_{2} \\
-h_{2}^{*} & h_{1}^{*}
\end{array}\right] \rightarrow\left[\begin{array}{cccc}
h_{1} & h_{2} & h_{3} & h_{4} \\
-h_{2}^{*} & h_{1}^{*} & -h_{4}^{*} & h_{3}^{*} \\
-h_{3}^{*} & -h_{4}^{*} & h_{1}^{*} & h_{2}^{*} \\
h_{4} & -h_{3} & -h_{2} & h_{1}
\end{array}\right]
$$

That is, the complex scalars $h_{1}$ and $h_{2}$ appearing to the left of the arrow " $\rightarrow$ " are replaced by the $2 \times 2$ matrices

$$
\begin{aligned}
& \mathbf{H}_{1}=\left[\begin{array}{cc}
h_{1} & h_{2} \\
-h_{2}^{*} & h_{1}^{*}
\end{array}\right], \\
& \mathbf{H}_{2}=\left[\begin{array}{cc}
h_{3} & h_{4} \\
-h_{4}^{*} & h_{3}^{*}
\end{array}\right],
\end{aligned}
$$

and then reinserted into the Alamouti space-time channel matrix

$$
\left[\begin{array}{cc}
\mathbf{H}_{1} & \mathbf{H}_{2} \\
-\mathbf{H}_{2}^{*} & \mathbf{H}_{1}^{*}
\end{array}\right]
$$

where $*$ denotes complex conjugation without transposition.

\footnotetext{
${ }^{2}$ The outage capacity of this scheme was originally reported in [19].
}

This results in the following symbol block $\mathbf{S}$ for transmitting the four symbols $\mathbf{s}=\left[s_{1}, \ldots, s_{4}\right]^{T}$ :

$$
\mathbf{S}=\left[\begin{array}{cccc}
s_{1} & s_{2} & s_{3} & s_{4} \\
s_{2}^{*} & -s_{1}^{*} & s_{4}^{*} & -s_{3}^{*} \\
s_{3}^{*} & s_{4}^{*} & -s_{1}^{*} & -s_{2}^{*} \\
s_{4} & -s_{3} & -s_{2} & s_{1}
\end{array}\right]
$$

The received vector can be expressed in the same form as (5). Converting the received vector by complex conjugation

$$
\begin{array}{ll}
y_{1}=r_{1}, & v_{1}=\bar{v}_{1}, \\
y_{2}=r_{2}^{*}, & v_{2}=\bar{v}_{2}^{*}, \\
y_{3}=r_{3}^{*}, & v_{3}=\bar{v}_{3}^{*}, \\
y_{4}=r_{4}, & v_{4}=\bar{v}_{4},
\end{array}
$$

results in the following equivalent transmission scheme:

$$
\mathbf{y}=\mathbf{H s}+\mathbf{v}
$$

in which $\mathbf{H}$ appears again as channel transmission matrix. If $\overline{\mathbf{v}}$ is a complex-valued Gaussian vector with i.i.d. elements, then so is $\mathbf{v}$.

\section{1. $M L$ receiver performance}

While a standard ML approach is possible with correspondingly high complexity, an alternative ML approach applying matched filtering is first possible with much less complexity. After the matched filtering operation, the resulting ma$\operatorname{trix} \mathbf{H}^{H}$ is

$$
\mathbf{G}=\mathbf{H}^{H} \mathbf{H}=\mathbf{H H}^{H}=h^{2}\left[\begin{array}{cc}
\mathbf{I}_{2} & X \mathbf{J}_{2} \\
-X \mathbf{J}_{2} & \mathbf{I}_{2}
\end{array}\right],
$$

where the $2 \times 2$ matrix

$$
\mathbf{J}_{2}=\left[\begin{array}{cc}
0 & 1 \\
-1 & 0
\end{array}\right]
$$

as well as the Grammian $\mathbf{G}$ have been introduced. The gain of the channel is

$$
h^{2}=\left|h_{1}\right|^{2}+\left|h_{2}\right|^{2}+\left|h_{3}\right|^{2}+\left|h_{4}\right|^{2}
$$

and the channel dependent real-valued random variable $X$ is defined as follows:

$$
X=\frac{2 \operatorname{Re}\left(h_{1} h_{4}^{*}-h_{2} h_{3}^{*}\right)}{h^{2}} .
$$

By applying the matched filter $\mathbf{H}^{H}$, this results in the reception of the following vector:

$$
\mathbf{z}=\mathbf{H}^{H} \mathbf{y}=\mathbf{H}^{H} \mathbf{H} \mathbf{s}+\mathbf{H}^{H} \mathbf{v}=h^{2}\left[\begin{array}{l}
s_{1}+X s_{4} \\
s_{2}-X s_{3} \\
s_{3}-X s_{2} \\
s_{4}+X s_{1}
\end{array}\right]+\mathbf{H}^{H} \mathbf{v}
$$


in which the pair $\left\{s_{1}, s_{4}\right\}$ is decoupled from $\left\{s_{2}, s_{3}\right\}$ allowing for a low-complexity solution based on the newly formed receiver vector $\mathbf{z}$.

The ML decoder selects s minimizing

$$
\Lambda_{1}(\mathbf{s})=\|\mathbf{y}-\mathbf{H s}\|^{2}=\mathbf{s}^{H} \mathbf{G s}-2 \operatorname{Re}\left(\mathbf{y}^{H} \mathbf{H s}\right)+\|\mathbf{y}\|^{2}
$$

for all permissible symbol vectors $\mathbf{s}$ from the transmitter alphabet and spatially white interference plus noise was assumed. Alternatively, the matched filter can be applied to $\mathbf{y}$ and the ML estimator can be implemented on its output $\mathbf{z}$ given in (20) leading to

$$
\Lambda_{2}(\mathbf{s})=(\mathbf{z}-\mathbf{G} \mathbf{s})^{H} \mathbf{G}^{-1}(\mathbf{z}-\mathbf{G} \mathbf{s})
$$

Note that it needs to be taken into account that the noise plus interference is spatially correlated after filtering. Assuming the elements $v_{k}$ of $\mathbf{v}$ to be zero mean and spatially white with variance $\sigma_{V}^{2}$ results in $\mathbf{w}=\mathbf{H}^{H} \mathbf{v}$ with covariance matrix

$$
\mathrm{E}\left[\mathbf{w} \mathbf{w}^{H}\right]=\sigma_{V}^{2} \mathbf{H}^{H} \mathbf{H}=\sigma_{V}^{2} \mathbf{G}
$$

The advantage of this approach is that this partly decouples the symbols. The pair $\left\{s_{1}, s_{4}\right\}$ is decoupled from $\left\{s_{2}, s_{3}\right\}$ allowing for a low-complexity ML receiver using the partial metrics

$$
\begin{aligned}
& \Lambda_{2 a}\left(s_{1}, s_{4}\right) \\
& =\left|z_{1}-h^{2}\left(s_{1}+X s_{4}\right)\right|^{2}+\left|z_{4}-h^{2}\left(s_{4}+X s_{1}\right)\right|^{2} \\
& \quad-2 X \operatorname{Re}\left\{\left[z_{1}-h^{2}\left(s_{1}+X s_{4}\right)\right]\left[z_{4}^{*}-h^{2}\left(s_{4}^{*}+X s_{1}^{*}\right)\right]\right\}, \\
& \Lambda_{2 b}\left(s_{2}, s_{3}\right) \\
& =\left|z_{2}-h^{2}\left(s_{2}-X s_{3}\right)\right|^{2}+\left|z_{3}-h^{2}\left(s_{3}-X s_{2}\right)\right|^{2} \\
& \quad+2 X \operatorname{Re}\left\{\left[z_{2}-h^{2}\left(s_{2}-X s_{3}\right)\right]\left[z_{3}^{*}-h^{2}\left(s_{3}^{*}-X s_{2}^{*}\right)\right]\right\} .
\end{aligned}
$$

Note that the two metrics $\Lambda_{2 a}$ and $\Lambda_{2 b}$ are positive definite when $|X|<1$. They become semidefinite for $|X|=1$. In UMTS with QPSK modulation, this requires a search over $2 \times 16$ vector symbols rather than over 256 .

\subsection{Performance of linear receivers}

Linear receivers typically suffer from noise enhancement. In this section, the increased noise caused by ZF and minimum mean squared error (MMSE) detectors is investigated. Both receivers can be described by the following detection principle:

$$
\hat{\mathbf{s}}=\left(\mathbf{H}^{H} \mathbf{H}+\mu \mathbf{I}_{4}\right)^{-1} \mathbf{z},
$$

where $\mu=0$ for ZF and $\mu=\sigma_{V}^{2}$ for MMSE. It turns out that both detection principles have essentially the same receiver complexity. The following lemmas can be stated.
Lemma 1. Given the $4 \times 4$ Alamouti scheme as described in (10), the eigenvalues of $\mathbf{H}^{H} \mathbf{H} / h^{2}$ are given by

$$
\lambda_{1}=\lambda_{2}=1+X, \quad \lambda_{3}=\lambda_{4}=1-X,
$$

where $h^{2}$ and $X$ are defined in (18) and (19).

Proof. The Grammian $\mathbf{H}^{H} \mathbf{H}$ is diagonalized by $\mathbf{V}_{4}^{T} \mathbf{H}^{H} \mathbf{H V}_{4}$ with the orthogonal matrix

$$
\mathbf{V}_{4}=\frac{1}{\sqrt{2}}\left[\begin{array}{ll}
\mathbf{I}_{2} & \mathbf{J}_{2} \\
\mathbf{J}_{2} & \mathbf{I}_{2}
\end{array}\right]
$$

Some favorable properties are worth mentioning. The eigenvectors of $\mathbf{H}^{H} \mathbf{H}$ which are stacked in the columns of $\mathbf{V}_{4}$ do not depend on the channel; they are constant. The scaled matrix $\sqrt{2} \mathbf{V}_{4}$ is sparse, that is, half of its elements vanish and the nonzero entries are \pm 1 .

Lemma 2. If the channel coefficients $h_{i}(i=1, \ldots, 4)$ are i.i.d. complex Gaussian variates with zero mean and variance 1/4, then the following properties hold:

(1) $X$ and $h^{2}$ are independent;

(2) let $\lambda_{i}$ be an eigenvalue of $\mathbf{H}^{H} \mathbf{H} / h^{2}$. The probability density of $\lambda_{i}$ is $f_{\lambda, 4}(\lambda)=(3 / 4) \lambda(2-\lambda)$ for $0<\lambda<2$ and zero elsewhere. Likewise, $\lambda_{i} / 2$ is beta(2,2)-distributed;

(3) let $\xi_{i}$ be an eigenvalue of $\mathbf{H}^{H} \mathbf{H}$. The probability density of $\xi_{i}$ is $f_{\xi}(\xi)=4 \xi e^{-2 \xi}$ for $\xi>0$.

Proof. The joint distribution of $X$ and $h^{2}$ is derived in Appendix A. The eigenvalues $\xi_{i}$ of $\mathbf{H}^{H} \mathbf{H}$ and $\lambda_{i}$ of $\mathbf{H}^{H} \mathbf{H} / h^{2}$ are proportional to each other, that is, $\xi_{i}=h^{2} \lambda_{i}$ for $i=$ $1, \ldots, 4$.

It can be concluded that $\mathrm{E}\left[\lambda_{i}\right]=1$ and $\operatorname{Var}\left(\lambda_{i}\right)=0.2$ for all $i$, indicating that the normalized channel matrix $\mathbf{H}^{H} \mathbf{H} / h^{2}$ is close to a unitary matrix with high probability.

Let $\gamma \geq 1$ be the following random variable which depends on the channel gain if $\mu>0$ :

$$
\gamma=\frac{h^{2}+\mu}{h^{2}}= \begin{cases}1 & \text { for ZF } \\ 1+\frac{\sigma_{V}^{2}}{h^{2}} & \text { for MMSE }\end{cases}
$$

For evaluating the BER of the linear receiver for general $\mu \neq$ 0 ,

$$
\begin{array}{r}
\operatorname{tr}\left[\left(\mathbf{H}^{H} \mathbf{H}+\mu \mathbf{I}_{4}\right)^{-1} \mathbf{H}^{H} \mathbf{H}\left(\mathbf{H}^{H} \mathbf{H}+\mu \mathbf{I}_{4}\right)^{-1}\right] \\
=\left(\frac{4}{h^{2}}\right) \frac{\gamma^{2}+X^{2}(1-2 \gamma)}{\left(\gamma^{2}-X^{2}\right)^{2}}
\end{array}
$$

needs to be evaluated which is obtained via

$$
\left[\mathbf{H}^{H} \mathbf{H}+\mu \mathbf{I}_{4}\right]^{-1}=\frac{1}{h^{2}\left(\gamma^{2}-X^{2}\right)}\left(\begin{array}{cc}
\gamma \mathbf{I}_{2} & -X \mathbf{J}_{2} \\
X \mathbf{J}_{2} & \gamma \mathbf{I}_{2}
\end{array}\right) .
$$

When replacing the arguments of the complementary error function with (29), two interpretations can be discussed. 
Comparing the arguments of the complementary error function with the standard ML solution for multiple diversity, one recognizes the beneficial diversity term $h^{2}$ indicating four times diversity together with an additional term, say

$$
\delta_{4} \triangleq \frac{\gamma^{2}+X^{2}(1-2 \gamma)}{\left(\gamma^{2}-X^{2}\right)^{2}}=\frac{1}{\gamma^{2}-X^{2}}-2(\gamma-1) \frac{X^{2}}{\left(\gamma^{2}-X^{2}\right)^{2}} .
$$

In Appendix A, it is shown that $X$ and $h^{2}$ are statistically independent variates. Therefore, $\delta_{4}$ can be interpreted as an increase in noise while $h^{2}$ causes full fourth-order diversity. Alternatively, one can interpret the whole expression $\alpha_{\mathrm{ZF}, 4}=h^{2} \delta_{4}$ as defining a new fading factor with the true diversity order without noise increase. Both interpretations can be used to describe the scheme's performance.

\subsubsection{Noise enhancement}

If the first interpretation is favoured, the following result is obtained.

Lemma 3. Given the $4 \times 4$ Alamouti scheme in independent flat Rayleigh fading as described in (10), a four-times diversity is obtained at the expense of a noise enhancement of

$$
\mathrm{E}\left[\delta_{4}\right]=\frac{3}{2}-2 \mu^{2}+2 \mu e^{2 \mu} \mathrm{E}_{1}(2 \mu)\left(2 \mu^{2}+\mu-2\right),
$$

where $\mathrm{E}_{n}(x)$ denotes the exponential integral defined for $\operatorname{Re}(x)$ $>0$ as follows:

$$
\mathrm{E}_{n}(x) \triangleq \int_{1}^{\infty} \frac{e^{-x t}}{t^{n}} d t
$$

Proof. The expectation E $\left[\delta_{4}\right]$ in (A.10) needs to be evaluated. Note that $\delta_{4}$ depends on $X$ and $h^{2}$. It is shown in Appendix A that $X$ and $h^{2}$ are independent if $h_{1}, \ldots, h_{4}$ are i.i.d. complexvalued zero-mean Gaussian variates. Therefore, we can evaluate $\mathrm{E}\left[\delta_{4}\right]$ via (A.11) which leads to the result (32).

In case of a ZF receiver, the noise is increased by a factor of $3 / 2$ which corresponds to $1.76 \mathrm{~dB}$, a value for which the four-times diversity scheme gives much better results as long as $E_{b} / N_{0}$ is larger than about $3 \mathrm{~dB}$. Therefore, the noise enhancement $\mathrm{E}\left[\delta_{4}\right]$ is maximum for ZF receivers $(\mu=0)$ and it does not exceed $1.76 \mathrm{~dB}$ for MMSE. The formula

$$
\mathrm{E}\left[\delta_{N_{T}}\right] \triangleq\left(\frac{1}{N_{T}}\right)^{2} \mathrm{E}\left[\operatorname{tr}\left(\left(\mathbf{H}^{H} \mathbf{H}\right)^{-1}\right) \operatorname{tr}\left(\mathbf{H}^{H} \mathbf{H}\right)\right]=2 \frac{N_{T}-1}{N_{T}}
$$

seems to describe the noise enhancement for ZF receivers for the general case of $N_{T}$ transmit antennas. Note that $\operatorname{tr}\left(\mathbf{H}^{H} \mathbf{H}\right)$ is the squared Frobenius norm of $\mathbf{H}$. The argument of the expectation operator is closely related to the numerical condition number $\kappa$ of $\mathbf{H}$. Let $\xi_{N_{T}}$ and $\xi_{1}$ be the largest and the smallest eigenvalue of $\mathbf{H}^{H} \mathbf{H}$, respectively. Then $\operatorname{tr}\left(\left(\mathbf{H}^{H} \mathbf{H}\right)^{-1}\right) \operatorname{tr}\left(\mathbf{H}^{H} \mathbf{H}\right) \geq \xi_{N_{T}} / \xi_{1}=\kappa^{2}$. The noise enhancement can be lower bounded by the squared numerical condition number, that is, $\mathrm{E}\left[\delta_{N_{T}}\right] \geq \mathrm{E}\left[\kappa^{2}\right]$.

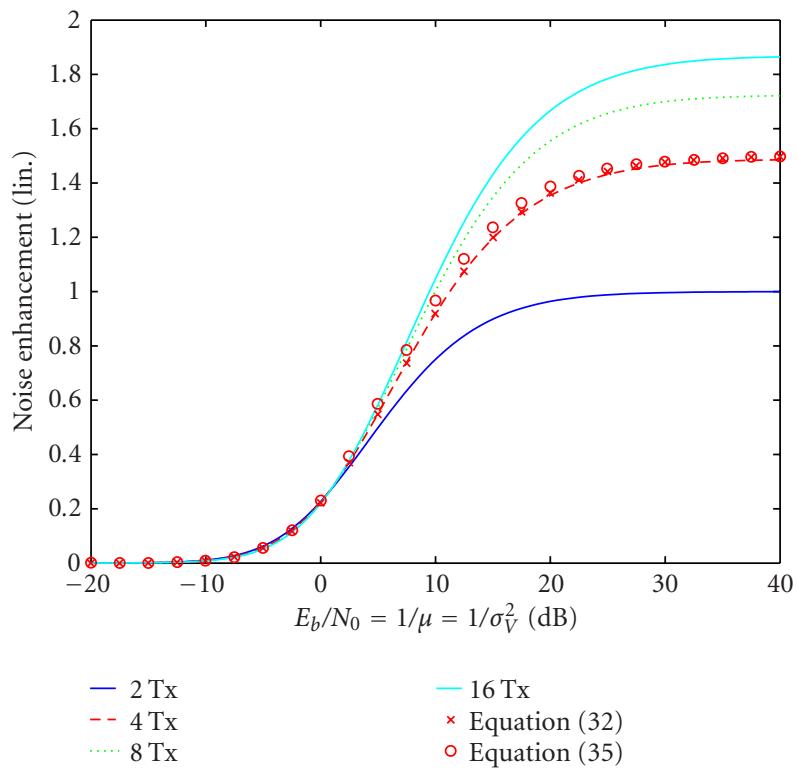

Figure 1: Comparison of the noise enhancement versus $E_{b} / N_{0}=$ $1 / \sigma_{V}^{2}$ for ZF and MMSE receivers.

The formula was explicitly validated for $N_{T}=2,4$, and 8 and with Monte Carlo simulations for larger values of $N_{T}$. Although no formal proof exists, the upper limit for the noise enhancement was found at $3 \mathrm{~dB}$. The behavior of (32) versus $1 / \mu$ (which equals $E_{b} / N_{0}$ for the MMSE) is shown in Figure 1 indicated by crosses labeled "x."

Additional insight into the behavior of (32) is gained by regarding the channel gain $h^{2}$ as approximately constant, an assumption that holds asymptotically true for $N_{T} \rightarrow \infty$. This assumption enables us to replace the joint expectation over $X$ and $\gamma$ in (32) by a conditional one, that is, conditioned on $h^{2}$,

$$
\mathrm{E}\left[\delta_{4} \mid h^{2}\right]=\frac{9}{2} \gamma-3+\left(\frac{9}{4} \gamma^{2}-\frac{3}{2} \gamma-\frac{3}{4}\right) \log \frac{\gamma-1}{\gamma+1} .
$$

This approximation is compared with the exact expression of (32) in Figure 1 where the approximation obtained from (35) is plotted versus $E[1 /(\gamma-1)]=E_{b} / N_{0}$. The values are indicated by circles labeled "॰." The horizontal shift in $E_{b} / N_{0}$ between (32) and (35) is generally less than $1 \mathrm{~dB}$. This approximation becomes exact for the case of $\mathrm{ZF}$ receiver where $\mu \rightarrow 0$, that is, the limit for $\gamma \rightarrow 1$ of (35) is $3 / 2$.

\subsubsection{True diversity}

The second interpretation of (29) leads to a refined diversity order. In this case, the term in $\gamma$ and $X$ purely modifies the diversity but leaves the noise part unchanged. The BER performance can be computed explicitly. We restrict ourselves to the ZF case for which $\gamma=1$ and $\delta_{4}=1 /\left[1-X^{2}\right]$. In this case, $\delta_{4}$ and $h^{2}$ are statistically independent. We obtain

$$
\mathrm{BER}_{\mathrm{ZF}}=\int_{h} \int_{\delta} \operatorname{erfc}\left(\frac{h^{2} \delta}{2 \sigma_{V}^{2}}\right) \frac{h^{3} e^{-h}}{2 \Gamma(4)} f_{\delta}(\delta) d h d \delta .
$$




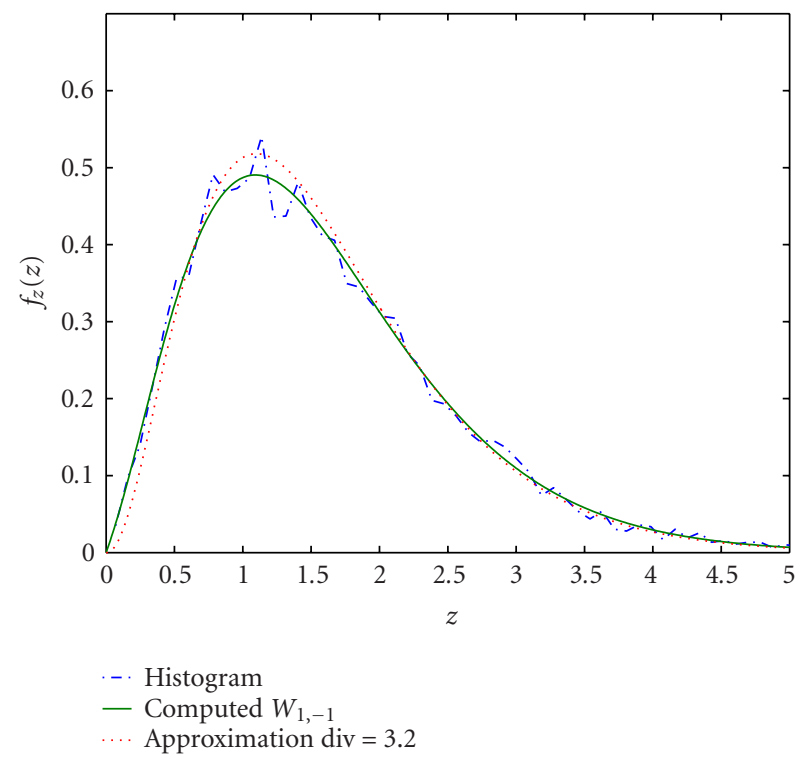

Figure 2: Histogram of a sample of $z$ defined in (40) and its density $f_{z}(z)$ in $(43)$.

Using the result from [13], (2) is obtained correspondingly, however, with a different solution for a random variable $\mu$ :

$$
\mu(X)=\sqrt{\frac{E_{b} / N_{0}}{2\left(1-X^{2}\right)+E_{b} / N_{0}}},
$$

leading to rather involved terms. A much simpler method is to interpret the term $h^{2} \delta$ as a new fading factor $\alpha_{\mathrm{ZF}, 4}$ with $\chi$-statistics. Since $\delta$ is a fractional number, the new factor $\alpha_{\mathrm{ZF}, 4}=h^{2} \delta$ cannot be expected to have an integer number of freedoms. Comparing with a Nakagami- $m$ density, the mean value of $h^{2} \delta$ corresponds to the number of degrees of freedom $m$ for this density. Computing $\mathrm{E}\left[h^{2} \delta_{4}\right]=m=3.2$ is obtained. Figure 2 displays a histogram of $\alpha_{\mathrm{ZF}, 4}$ from 5,000 runs. Furthermore, the exact density function is shown and a close fit obtained by the squared Nakagami- $m$ distribution with $m=3.2$, or equivalent $\chi^{2}$ with 6.4 degrees of freedom. This result contradicts the general belief that ZF receivers obtain only $2\left(N_{R}-N_{T}+1\right)=2$ degrees of freedom. The result is different here due to the channel structuring.

An exact derivation of the probability density for this random variable is lengthy and is only sketched here. The random variable $\left(1-X^{2}\right) h^{2}$ can be constructed from two independent variables $\mathbf{u}^{H} \mathbf{u}$ and $\mathbf{v}^{H} \mathbf{v}$ which are each $\chi^{2}$ distributed with four degrees of freedom (diversity order two). Substitute

$$
\mathbf{x}^{T}=\left[h_{1}, h_{2}\right], \quad \mathbf{y}^{T}=\left[h_{4},-h_{3}\right] .
$$

Then $X=\left(\mathbf{x}^{H} \mathbf{y}+\mathbf{y}^{H} \mathbf{x}\right) /\left(\mathbf{x}^{H} \mathbf{x}+\mathbf{y}^{H} \mathbf{y}\right)$. Using $\mathbf{u}=[\mathbf{x}-\mathbf{y}] / \sqrt{2}$ and $\mathbf{v}=[\mathbf{x}+\mathbf{y}] / \sqrt{2}$, the following result is obtained:

$$
\left(1-X^{2}\right) h^{2}=4 \frac{\mathbf{u}^{H} \mathbf{u} \mathbf{v}^{H} \mathbf{v}}{\mathbf{u}^{H} \mathbf{u}+\mathbf{v}^{H} \mathbf{v}}=\frac{4}{1 / \mathbf{u}^{H} \mathbf{u}+1 / \mathbf{v}^{H} \mathbf{v}} .
$$

The joint density $p_{w, z}(w, z)$ of this expression can be computed via the transformation

$$
z=\frac{1}{1 / \mathbf{u}^{H} \mathbf{u}+1 / \mathbf{v}^{H} \mathbf{v}}, \quad w=\mathbf{v}^{H} \mathbf{v}
$$

achieving

$$
p_{w, z}(w, z)=\frac{w^{3} z}{(w-z)^{3}} \exp \left(-\frac{w^{2}}{w-z}\right) .
$$

The density of $z$ is found by marginalizing the joint density $p(w, z)$. The density can be expressed using a Whittaker function (see [20]):

$$
\begin{aligned}
f_{z}(z) & =2^{9} z \int_{u}^{\infty} \frac{t^{3 / 2} \exp (-4 t)}{\sqrt{t-z}} d t \\
& =2^{6} z^{3 / 2} \Gamma\left(\frac{1}{2}\right) \exp (-2 z) W_{1,-1}(4 z) \approx \frac{4^{3.2} z^{2.2} e^{4 z}}{\Gamma(3.2)} .
\end{aligned}
$$

This last approximation is also shown in Figure 2, obviously a good fit.

\subsection{Simulation results}

Figure 3 displays the simulated behavior of the uncoded BER transmitting QPSK (gray coded) of the linear MMSE receiver and zero fading correlation between the four transmit paths. The BER results were averaged over 16,000 symbols and 3,200 selections of channel matrices $\mathbf{H}$ for each simulated $E_{b} / N_{0}$. For comparison, the BER from the $\mathrm{ZF}$ receiver and the cases of ideal two- and four-path diversity are also shown. The values marked by circles "。” labeled "expected theory" are the same as for four-path diversity, but shifted by the noise enhancement (n.e.) of $1.76 \mathrm{~dB}$. Compared to the $\mathrm{ZF}$ receiver performance, there is just a little improvement for MMSE.

For practical considerations, it is of interest to investigate the performance when the four paths are correlated, as can be expected in a typical transmission environment. Figure 4 displays the situation when the antenna elements are correlated by a factor of $\{0.5,0.75,0.95\}$. As the figure reveals, no further loss is shown until the value exceeds 0.5 . Only with very strong correlation (0.95), a degradation of $4 \mathrm{~dB}$ was noticed.

\subsection{Diversity cumulating property of receive antennas}

An interesting property is worth mentioning coming with the $4 \times 1$ extended Alamouti scheme when using more than one receive antenna. Typically adding more receive antennas gives rise to expect a higher diversity order in the transmission system, however, available only at the expense of more complexity in the receiver algorithms. In the extended Alamouti scheme, the behavior is slightly different as stated in the following lemma.

Lemma 4. When utilizing an arbitrary number $N_{R}$ of receive antennas, the extended Alamouti scheme can obtain an $N_{R}$-fold 


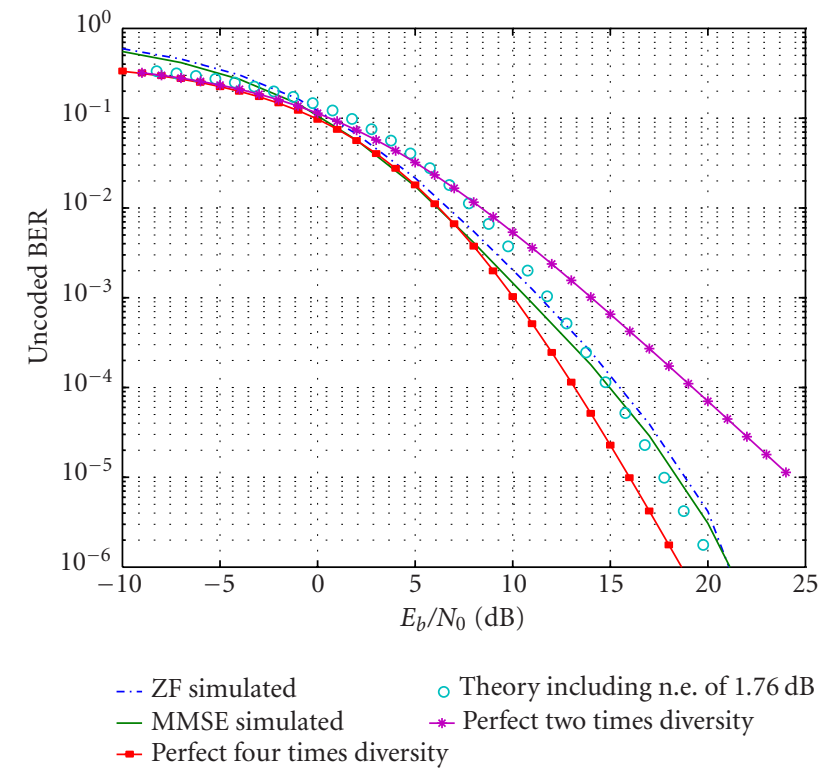

FIGURE 3: BER for four-antenna scheme with linear MMSE receiver and zero correlation between antennas.

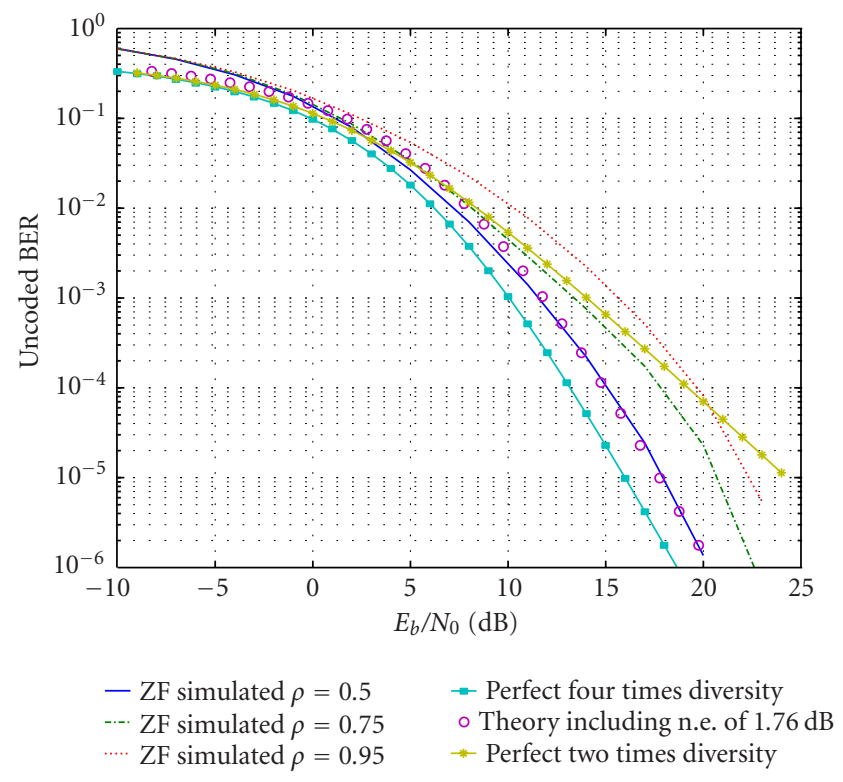

FIGURE 4: BER for four-antenna scheme with ZF receiver, fading correlation between adjacent antenna elements is $\{0.5,0.75,0.95\}$.

diversity compared to the single receive antenna case requiring only an asymptotically linear complexity $O\left(N_{R}\right)$ for $M L$ as well as linear receivers.

Proof. The proof will be shown for two receive antennas. Extending it to more than two is a straight forward exercise:

$$
\mathbf{r}_{1}=\mathbf{H}_{1} \mathbf{s}+\mathbf{v}_{1} ; \quad \mathbf{r}_{2}=\mathbf{H}_{2} \mathbf{s}+\mathbf{v}_{2}
$$

Matched filtering can be applied and the corresponding terms are summed up to obtain

$$
\begin{aligned}
\mathbf{H}_{1}^{H} \mathbf{r}_{1}+\mathbf{H}_{2}^{H} \mathbf{r}_{2} & =\left[\mathbf{H}_{1}^{H} \mathbf{H}_{1}+\mathbf{H}_{2}^{H} \mathbf{H}_{2}\right] \mathbf{s}+\mathbf{H}_{1}^{H} \mathbf{v}_{1}+\mathbf{H}_{2}^{H} \mathbf{v}_{2} \\
& =\left[\mathbf{H}_{1}^{H} \mathbf{H}_{1}+\mathbf{H}_{2}^{H} \mathbf{H}_{2}\right] \mathbf{s}+\tilde{\mathbf{v}} .
\end{aligned}
$$

Note that the new matrix $\left[\mathbf{H}_{1}^{H} \mathbf{H}_{1}+\mathbf{H}_{2}^{H} \mathbf{H}_{2}\right]$ preserves the form (16):

$$
\mathbf{H}_{1}^{H} \mathbf{H}_{1}+\mathbf{H}_{2}^{H} \mathbf{H}_{2}=\left(h_{1}^{2}+h_{2}^{2}\right)\left[\begin{array}{cccc}
1 & 0 & 0 & X \\
0 & 1 & -X & 0 \\
0 & -X & 1 & 0 \\
X & 0 & 0 & 1
\end{array}\right],
$$

with $X=\left[X_{1} h_{1}^{2}+X_{2} h_{2}^{2}\right] /\left[h_{1}^{2}+h_{2}^{2}\right]$. Thus, the matrix maintains its form and therefore, complexity of ML or a linear receiver remains identical to the one antenna case. Only the matched filtering needs to be performed additionally for as many receive antennas are present. The leading term $h_{1}^{2}+h_{2}^{2}$ describes the diversity order, being twice as high as before. For $N_{R}$ receiver antennas, a sum of all terms $h_{k}^{2}, k=1, \ldots, N_{R}$, will appear in this position indicating an $N_{R}$-fold increase in capacity.

Note that $N_{R}$ receiver antennas can be purely virtual and do not necessarily require a larger RF front end effort. For example, UMTS's WCDMA scheme enables RAKE techniques to be utilized. Thus, at tap delays $\tau_{k}$ where large energies occur, a finger of the RAKE receiver is positioned. Correspondingly, the channel matrix $\mathbf{H}$ consists in this case of several components, all located at $K$ different delay times. The received values can be structured in one vector as well and $\mathbf{y}=\mathbf{H s}+\mathbf{v}$ is obtained again, however now with $\mathbf{y}$ is of dimension $4 K \times 1$ and $\mathbf{H}$ of dimension $4 K \times 4$, while $\mathbf{s}$ remains of dimension $4 \times 1$ as before. The previously discussed schemes can be applied as well and each term $h^{2}$ now consists of $K$ times as many components as before, thus increasing diversity by a factor of $K$. In conclusion, such techniques work as well in a scenario with interchip interference as in flat Rayleigh fading with the additional benefit of having even more diversity and thus a better QoS, provided the crosscorrelation between different users remains limited.

\section{EIGHT AND MORE ANTENNA SCHEMES}

Applying (10) several times ( $m-1$ times), solutions for $N_{T}=2^{m} \times 1$ antenna schemes can be obtained. The obtained matrices exhibit certain properties that will be utilized in the following. They are listed in the following lemma and proven in Appendix B.

Lemma 5. Applying rule (10) $m-1$ times results in matrices $\mathbf{H}$ of dimension $N_{T} \times N_{T}, N_{T}=2^{m}$, with the following properties:

(1) all entries of $\mathbf{H}^{H} \mathbf{H}$ are real-valued;

(2) the matrix $\mathbf{H}^{H} \mathbf{H}$ is of the form

$$
\mathbf{H}^{H} \mathbf{H}=\left[\begin{array}{cc}
\mathbf{A} & \mathbf{B} \\
-\mathbf{B} & \mathbf{A}
\end{array}\right]
$$


and the inverse of $\mathbf{H}^{H} \mathbf{H}$ is of block matrix form

$$
\left[\mathbf{H}^{H} \mathbf{H}\right]^{-1}=\left[\begin{array}{cc}
\mathbf{A} & -\mathbf{B} \\
\mathbf{B} & \mathbf{A}
\end{array}\right]\left[\begin{array}{cc}
\left(\mathbf{A}^{2}+\mathbf{B}^{2}\right)^{-1} & \varnothing \\
\varnothing & \left(\mathbf{A}^{2}+\mathbf{B}^{2}\right)^{-1}
\end{array}\right] .
$$

Due to the form (47), all eigenvalues are double; ${ }^{3}$

(3) each nondiagonal entry $X_{i}$ of $\mathbf{H}^{H} \mathbf{H} / \operatorname{tr}\left[\mathbf{H}^{H} \mathbf{H}\right]$ is either zero, or $X_{i}$ follows the distribution

$$
f_{X}(\xi)=\frac{1}{2^{N_{T}-2} \mathrm{~B}\left(N_{T} / 2, N_{T} / 2\right)}\left(1-\xi^{2}\right)^{N_{T} / 2-1}, \quad|\xi| \leq 1 .
$$

Applying rule (10) two times in succession results in the $8 \times 8$ scheme. It can immediately be verified that the matrix $\mathbf{H}^{H} \mathbf{H}$ is given by

$$
\mathbf{H}^{H} \mathbf{H}=h^{2}\left[\begin{array}{cccc}
\mathbf{I}_{2} & X \mathbf{J}_{2} & -Z \mathbf{J}_{2} & Y \mathbf{I}_{2} \\
-X \mathbf{J}_{2} & \mathbf{I}_{2} & -Y \mathbf{I}_{2} & -Z \mathbf{J}_{2} \\
Z \mathbf{J}_{2} & -Y \mathbf{I}_{2} & \mathbf{I}_{2} & X \mathbf{J}_{2} \\
Y \mathbf{I}_{2} & Z \mathbf{J}_{2} & -X \mathbf{J}_{2} & \mathbf{I}_{2}
\end{array}\right] \text {, }
$$

with

$$
\begin{aligned}
h^{2} & =\sum_{k=1}^{8}\left|h_{k}\right|^{2}, \\
X & =\frac{2 \operatorname{Re}\left(h_{1} h_{4}^{*}-h_{2} h_{3}^{*}+h_{5} h_{8}^{*}-h_{6} h_{7}^{*}\right)}{h^{2}}, \\
Y & =\frac{2 \operatorname{Re}\left(h_{1} h_{7}^{*}-h_{3} h_{5}^{*}+h_{2} h_{8}^{*}-h_{4} h_{6}^{*}\right)}{h^{2}}, \\
Z & =\frac{2 \operatorname{Re}\left(h_{2} h_{5}^{*}-h_{1} h_{6}^{*}+h_{4} h_{7}^{*}-h_{3} h_{8}^{*}\right)}{h^{2}} .
\end{aligned}
$$

According to property (2), the block structure of this matrix can be recognized. Note that $\mathbf{A}^{2}+\mathbf{B}^{2}=\alpha \mathbf{I}_{4}+\beta \mathbf{J}_{4}$, with

$$
\begin{gathered}
\mathbf{J}_{4}=\left[\begin{array}{cc}
\varnothing & \mathbf{J}_{2} \\
-\mathbf{J}_{2} & \varnothing
\end{array}\right], \\
\alpha=X^{2}-Y^{2}-Z^{2}+1, \quad \beta=2(X-Y Z),
\end{gathered}
$$

and the inverse can also be expressed by a combination of $\mathbf{I}_{4}$ and $\mathbf{J}_{4}$ :

$$
\left[\mathbf{A}^{2}+\mathbf{B}^{2}\right]^{-1}=\frac{1}{\alpha^{2}-\beta^{2}}\left(\alpha \mathbf{I}_{4}-\beta \mathbf{J}_{4}\right)
$$

if $|\alpha| \neq|\beta|$ which enables a computationally efficient implementation.

The ML receiver decouples into two $4 \times 4$ schemes by exploiting the structure of these matrices, (cf. Section 3.1). For UMTS with QPSK modulation, this leads to a search over $2 \times 256$ vector symbols rather than $4^{8}=65536$.

\footnotetext{
${ }^{3}$ The proof of the latter statement is simple: if an eigenvector $[\mathbf{x}, \mathbf{y}]$ exists for an eigenvalue $\lambda$, then also $[\mathbf{y},-\mathbf{x}]$ must be an eigenvector, linear independent of the first one, and thus the eigenvalues must be double.
}

\subsection{Performance of linear receivers}

Proceeding analogously to Section 3.2, the noise enhancement $\mathrm{E}\left[\delta_{8}\right]$ for the eight-antenna scheme is governed by $\operatorname{tr}\left[\left(\mathbf{H}^{H} \mathbf{H}+\mu \mathbf{I}_{8}\right)^{-1} \mathbf{H}^{H} \mathbf{H}\left(\mathbf{H}^{H} \mathbf{H}+\mu \mathbf{I}_{8}\right)^{-1}\right]=8 \delta_{8} / h^{2}$, where $\gamma=1+\mu / h^{2}$ and

$$
\delta_{8} \triangleq \frac{1}{8} \sum_{i=1}^{8} \frac{\lambda_{i}}{\left(\gamma+\lambda_{i}-1\right)^{2}}
$$

Lemma 6. All eigenvalues $\lambda_{i}$ of $\mathbf{H}^{H} \mathbf{H} / h^{2}$ in (50) are given by

$$
\begin{aligned}
& \lambda_{1}=\lambda_{2}=(1-X)+(Y-Z), \\
& \lambda_{3}=\lambda_{4}=(1+X)-(Y+Z), \\
& \lambda_{5}=\lambda_{6}=(1+X)+(Y+Z), \\
& \lambda_{7}=\lambda_{8}=(1-X)-(Y-Z) .
\end{aligned}
$$

Proof. The Grammian $\mathbf{H}^{H} \mathbf{H}$ is diagonalized by $\mathbf{V}_{8}^{T} \mathbf{H}^{H} \mathbf{H V}_{8}$ with the orthogonal matrix

$$
\mathbf{V}_{8}=\frac{1}{2}\left[\begin{array}{cccc}
\mathbf{I}_{2} & \mathbf{J}_{2} & \mathbf{J}_{2} & \mathbf{I}_{2} \\
\mathbf{J}_{2} & \mathbf{I}_{2} & -\mathbf{I}_{2} & -\mathbf{J}_{2} \\
\mathbf{J}_{2} & \mathbf{I}_{2} & \mathbf{I}_{2} & \mathbf{J}_{2} \\
-\mathbf{I}_{2} & -\mathbf{J}_{2} & \mathbf{J}_{2} & \mathbf{I}_{2}
\end{array}\right]
$$

resulting in the above given eigenvalues.

Lemma 7. If the channel coefficients $h_{i}(i=1, \ldots, 8)$ are i.i.d. complex-valued Gaussian variates with zero mean and variance $1 / 8$, then the following properties hold:

(1) let $\lambda_{i}$ be an eigenvalue of $\mathbf{H}^{H} \mathbf{H} / h^{2}$. The probability density of $\lambda_{i}$ is $f_{\lambda, 8}(\lambda)=(21 / 8192) \lambda(4-\lambda)^{5}$ for $0<$ $\lambda<4$ and zero elsewhere. Likewise, $\lambda_{i} / 4$ is beta $(2,6)-$ distributed;

(2) let $\xi_{i}$ be an eigenvalue of $\mathbf{H}^{H} \mathbf{H}$. The probability density of $\xi_{i}$ is $f_{\xi}(\xi)=4 \xi e^{-2 \xi}$ for $\xi>0$ and zero elsewhere.

Proof. It is sufficient to give the proof for one eigenvalue, say $\lambda_{5}$. The proof for the remaining eigenvalues proceeds similarly. By completing the squares (as in Appendix A), $h^{2} \lambda_{5} / 4$ can be regarded as the sum of two $\chi_{n}^{2}$-distributed variables with $n=2$ degrees of freedom each, that is,

$$
\left|\frac{h_{1}+h_{4}-h_{6}+h_{7}}{2}\right|^{2}+\left|\frac{h_{2}-h_{3}+h_{5}+h_{8}}{2}\right|^{2}
$$

By introducing an orthogonal transformation via the matrix $\mathbf{V}_{8}^{T}$ from (56), the proof is completed following the procedure in Appendices A and B.

The noise enhancement for the eight-antenna case and a ZF receiver $(\mu=0)$ is evaluated by using the eigenvalue statistics from Lemma 7 :

$$
\mathrm{E}\left[\delta_{8}\right]=\int_{0}^{4} \lambda^{-1} f_{\lambda, 8}(\lambda) d \lambda=\frac{7}{4}=1.75
$$




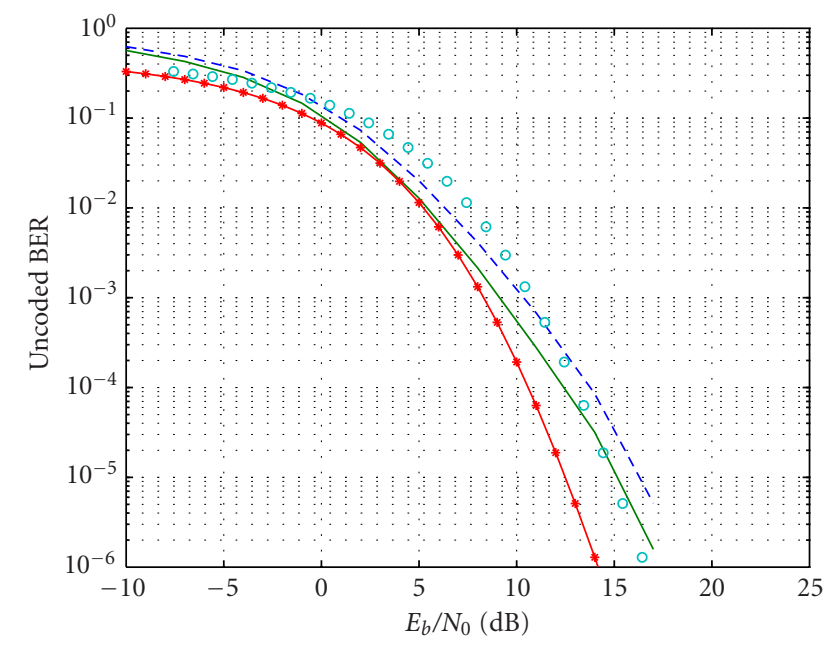

- - Eight-antenna scheme: ZF simulated

- Eight-antenna scheme: MMSE simulated

*- Perfect eight times diversity

- Theory including n.e. of $2.43 \mathrm{~dB}$

FIGURE 5: BER for eight-antenna scheme for ZF and MMSE receivers compared to theory.

or around $2.43 \mathrm{~dB}$. The noise enhancement for the general linear receiver $(\mu \geq 0)$ is obtained similarly to the fourantenna scheme; the result is

$$
\mathrm{E}\left[\delta_{8}\right]=\frac{7}{4}+2 \mu-\mu^{2}+\mu e^{2 \mu} \mathrm{E}_{1}(2 \mu)\left(2 \mu^{2}-3 \mu-6\right) .
$$

Thus, the noise enhancement of the MMSE receiver is always smaller than $2.43 \mathrm{~dB}$. Figure 1 compares the noise enhancement versus SNR for the ZF and MMSE receivers and for Alamouti's two-, and the proposed four-, and eight-antenna schemes. The noise enhancement for each scheme is calculated numerically by averaging over 4000 realizations of the channel matrix $\mathbf{H}$. For each realization, the eigenvalues $\lambda_{i}$ of $\mathbf{H}^{H} \mathbf{H}$ are numerically computed and subsequently averaged over $\left(h^{2} / N_{T}\right) \sum_{i=1}^{N_{T}} \lambda_{i} /\left(\lambda_{i}+\mu\right)^{2}$, where $N_{T}=2,4,8$, or 16 . The resulting averaged curves are shown in Figure 1 labeled "2 Tx," "4 Tx," and so forth.

The theoretical values marked by small crosses, labeled "x," are calculated according to $(32)$ versus $E_{b} / N_{0}=1 / \sigma_{V}^{2}=$ $1 / \mu$ for the MMSE case. The values marked by small circles, labeled "॰," are calculated according to the approximation in (35) versus $E_{b} / N_{0}=\mathrm{E}[1 /(\gamma-1)]$.

\subsection{Simulation results}

Figure 5 displays the simulated behavior of the uncoded BER for QPSK modulation and zero-fading correlation between the eight transmit paths. The BER results were averaged over 12,800 symbols and 4,000 selections of channel matrices $\mathbf{H}$ for each simulated $E_{b} / N_{0}$. The results are shown for a significance level of $99.7 \%$. In other words, the scheme assumes a tolerated outage probability of $0.3 \%$. Outage is assumed to occur if the numerical condition of $\mathbf{H}^{H} \mathbf{H}$ which is the ratio of the largest to the smallest eigenvalue exceeds $100 \approx 2^{7}$. In- verting these rare but adverse (nearly singular) channel matrices $\mathbf{H}^{H} \mathbf{H}$ lead to the loss of at least seven bits of numerical accuracy in the receiver. The values marked by little circles "o" labeled "expected theory" are the same as for eight-path diversity, but shifted by the noise variance increase of $2.43 \mathrm{~dB}$.

\section{ALAMOUTIZATION}

So far, mostly $N_{T} \times 1$ antenna schemes have been considered. However, in the future several antennas are likely to occur at the receiver as well. A cellular phone can carry two and a laptop as many as four antennas [17]. The proposed schemes can be applied, however, it remains unclear how to combine the received signals in an optimal fashion. In the following, an interesting approach is presented allowing an increase in diversity when the number of receiver antennas is more than one but typically less than the number of transmit antennas. The proposed STC schemes preserve a large part of the orthogonality so that the receivers can be implemented with low-complexity. The diversity is exploited in full and the noise enhancement remains small.

Proposition 2. Assume that a block matrix form of the channel matrix $\mathbf{H}$ is given by

$$
\mathbf{H}=\left[\mathbf{H}_{1} \mathbf{H}_{2}\right],
$$

where the matrices $\left\{\mathbf{H}_{1}, \mathbf{H}_{2}\right\}$ are not necessarily quadratic. Then, the scheme can be Alamouted by performing the following operation:

$$
\mathbf{G}=\left[\begin{array}{cc}
\mathbf{H}_{1} & \mathbf{H}_{2} \\
-\mathbf{H}_{2}^{*} & \mathbf{H}_{1}^{*} \\
\mathbf{H}_{2}^{*} & \mathbf{H}_{1}^{*} \\
\mathbf{H}_{1} & -\mathbf{H}_{2}
\end{array}\right] .
$$

At the receiver, a ZF operation is performed, obtaining the corresponding term $\mathbf{G}^{H} \mathbf{G}$ with the property

$$
\mathbf{G}^{H} \mathbf{G}=2\left[\begin{array}{cc}
\mathbf{H}_{1}^{H} \mathbf{H}_{1}+\mathbf{H}_{2}^{T} \mathbf{H}_{2}^{*} & \varnothing \\
\varnothing & \mathbf{H}_{1}^{T} \mathbf{H}_{1}^{*}+\mathbf{H}_{2}^{H} \mathbf{H}_{2}
\end{array}\right] .
$$

Thus perfect orthogonality on the nondiagonal block entries is achieved indicating little noise enhancement while the diagonal block terms indicate high diversity values. ${ }^{4}$

Example 1. A two-transmit-two-receive antenna system is considered:

$$
\mathbf{H}_{1}=\left[\begin{array}{l}
h_{1} \\
h_{2}
\end{array}\right], \quad \mathbf{H}_{2}=\left[\begin{array}{l}
h_{3} \\
h_{4}
\end{array}\right] .
$$

The matrix $\mathbf{G}^{H} \mathbf{G}$ becomes

$$
\mathbf{G}^{H} \mathbf{G}=2\left(\left|h_{1}\right|^{2}+\left|h_{2}\right|^{2}+\left|h_{3}\right|^{2}+\left|h_{4}\right|^{2}\right)\left[\begin{array}{ll}
1 & 0 \\
0 & 1
\end{array}\right] \text {. }
$$

\footnotetext{
${ }^{4}$ This was proposed in [4] in a simpler form.
} 
Thus, the full four times diversity can be explored, without a matrix inverse computation. Note that in this case, the transmit sequence at the two antennas reads

$$
\left\{\begin{array}{cccccccc}
s_{1} & s_{2} & -s_{3}^{*} & -s_{4}^{*} & s_{3}^{*} & s_{4}^{*} & s_{1} & s_{2} \\
s_{3} & s_{4} & s^{*} & s_{2}^{*} & s_{1}^{*} & s_{2}^{*} & -s_{3} & -s_{4}
\end{array}\right\} .
$$

Note also that during eight time periods, only four symbols are transmitted, that is, this particular scheme has the drawback of offering only half the symbol rate!

Example 2. Consider a $4 \times 2$ transmission scheme. The matrices are identified to

$$
\mathbf{H}_{1}=\left[\begin{array}{ll}
h_{11} & h_{12} \\
h_{21} & h_{22}
\end{array}\right], \quad \mathbf{H}_{2}=\left[\begin{array}{ll}
h_{13} & h_{14} \\
h_{23} & h_{24}
\end{array}\right] .
$$

The matrix $\mathbf{G}^{H} \mathbf{G}$ consists of two block matrices of size $2 \times 2$ on the diagonal. Thus, the scheme is still rather simple since only a $2 \times 2$ matrix has to be inverted although a four-path diversity is achieved. A comparison of the noise enhancement shows that for this $4 \times 2$ antenna system, $3 \mathrm{~dB}$ is gained compared to the $4 \times 1$ antenna system. Note that now the data rate is at full speed!

Example 3. The previously discussed $4 \times 1$ antenna system can be obtained when setting

$$
\mathbf{H}_{1}=\left[\begin{array}{ll}
h_{1} & h_{2}
\end{array}\right], \quad \mathbf{H}_{2}=\left[\begin{array}{ll}
h_{3} & h_{4}
\end{array}\right] .
$$

The reader may also try schemes in which the number of receive antennas is not given by $N_{R}=2^{n}$. As long as $N_{R}$ is even, the scheme can be separated in two matrices $\mathbf{H}_{1}$ and $\mathbf{H}_{2}$ of same size allowing the Alamoutization rule (Proposition 2) to be applied.

\section{COMBINING BLAST AND ALAMOUTI SCHEMES}

Although the proposed extended Alamouti schemes allow for utilizing the channel diversity without sacrificing the receiver complexity, not much has been said on data rates yet. In the case of $N_{T} \times 1$ antenna schemes, the $N_{T}$ symbols were repeated $N_{T}$ times in a different and specific order guaranteeing a data rate of one. Thus, the data rates in the proposed schemes typically remain constant (equal to one) when the schemes are quadratic and can be lower when the receive antenna number is smaller than the transmit antennas as pointed out in the previous section. In BLAST transmissions, this is different. In its simplest form, the V-BLAST coding [21], $N_{T}$ new symbols are offered to the $N_{T}$ transmit antennas at every symbol time instant thus achieving data rates $N_{T}$ times higher than in the Alamouti schemes. A combination of schemes can be achieved by simply transmitting more or less of the different repetitive transmissions. By utilizing the obtained transmission matrix structures, the diversity inherent in the transmission scheme can be exploited differently offering a trade-off between data rate and diversity order. In order to clarify this statement, an example is presented.
TABLE 1

\begin{tabular}{ccc}
\hline Antenna & $n=1$ & $n=2$ \\
\hline 1 & $s_{1}$ & $s_{2}^{*}$ \\
2 & $s_{2}$ & $-s_{1}^{*}$ \\
3 & $s_{3}$ & $s_{4}^{*}$ \\
4 & $s_{4}$ & $-s_{3}^{*}$ \\
\hline
\end{tabular}

Example 4. A $4 \times 2$ antenna scheme is considered for transmission. In a flat-fading channel system, eight Rayleigh coefficients are available describing the transmissions from the four transmit to the two receive antennas, the transmission matrix being

$$
\mathbf{H}=\left[\begin{array}{llll}
h_{11} & h_{12} & h_{13} & h_{14} \\
h_{21} & h_{22} & h_{23} & h_{24}
\end{array}\right] .
$$

It should thus be possible to transmit either four times the symbol data rate with diversity gain two, or two times the data rate with diversity four, or only at the symbol data rate but with diversity gain eight. In the first case, the $4 \times 1$ scheme as proposed in Section 3 will be used, repeating the four symbols four times, resulting in the reception of eight symbols. When assigning two paths each to one $2 \times 2$ matrix $\mathbf{H}_{i}$, $i=1, \ldots, 4$, the following transmission matrix is obtained:

$$
\mathbf{H}=\left[\begin{array}{cc}
\mathbf{H}_{1} & \mathbf{H}_{2} \\
-\mathbf{H}_{2}^{*} & \mathbf{H}_{1}^{*} \\
\mathbf{H}_{3} & \mathbf{H}_{4} \\
-\mathbf{H}_{4}^{*} & \mathbf{H}_{3}^{*}
\end{array}\right]
$$

Computing $\mathbf{H}^{H} \mathbf{H}$, a $4 \times 4$ matrix is obtained in a similar way to the $4 \times 1$ antenna case, however with twice the diversity. Thus in this case, a diversity of eight is achieved with a data rate of one.

On the other hand, by transmitting the sequences only twice, according to Table 1, the received signals at the two antennas can be formed to

$$
\left[\begin{array}{l}
y_{11} \\
y_{12} \\
y_{21} \\
y_{22}
\end{array}\right]=\left[\begin{array}{cccc}
h_{11} & h_{12} & h_{13} & h_{14} \\
-h_{12}^{*} & h_{11}^{*} & -h_{14}^{*} & h_{13}^{*} \\
h_{21} & h_{22} & h_{23} & h_{24} \\
-h_{22}^{*} & h_{21}^{*} & -h_{24}^{*} & h_{23}^{*}
\end{array}\right]\left[\begin{array}{l}
s_{1} \\
s_{2} \\
s_{3} \\
s_{4}
\end{array}\right]=\text { Hs. }
$$

Thus, computing $\mathbf{H}^{H} \mathbf{H}$ results simply in the following block matrix:

$$
\mathbf{H}^{H} \mathbf{H}=\left[\begin{array}{cc}
\gamma_{1} \mathbf{I} & \mathbf{B} \\
\mathbf{B}^{H} & \gamma_{2} \mathbf{I}
\end{array}\right]
$$

with $\gamma_{1}=\left|h_{11}\right|^{2}+\left|h_{12}\right|^{2}+\left|h_{13}\right|^{2}+\left|h_{14}\right|^{2}$ and $\gamma_{2}=\left|h_{21}\right|^{2}+$ $\left|h_{22}\right|^{2}+\left|h_{23}\right|^{2}+\left|h_{24}\right|^{2}$. Due to the condition $\mathbf{B}^{H} \mathbf{B}=\mathbf{B B}^{H}$, such matrices can be inverted with a $2 \times 2$ matrix inversion rather than a $4 \times 4$ :

$$
\left[\mathbf{H}^{H} \mathbf{H}\right]^{-1}=\left[\begin{array}{cc}
\gamma_{2} \mathbf{I} & -\mathbf{B} \\
-\mathbf{B}^{H} & \gamma_{1} \mathbf{I}
\end{array}\right]\left[\begin{array}{ll}
\mathbf{C} & \varnothing \\
\varnothing & \mathbf{C}
\end{array}\right]
$$

with $\mathbf{C}=\left[\gamma_{1} \gamma_{2} \mathbf{I}-\mathbf{B B}^{H}\right]^{-1}$. Thus, the underlying Alamouti scheme gives us the advantage of lower complexity while the 
BLAST scheme offers higher data rate. This specific scheme was investigated in $[22,23]$, where a diversity factor of six was found to closely match the diversity gain and the corresponding unitary matrices to diagonalize the scheme are presented.

Finally, the third transmission mode would send only one set of four symbols to the four transmit antennas. The corresponding matrix $\mathbf{H}^{H} \mathbf{H}$ is not of full rank and therefore, cannot be inverted. The entries on its diagonal consist of two times diversity terms like $\left|h_{11}\right|^{2}+\left|h_{12}\right|^{2}$. The decoding can be performed either in MMSE mode or with an ML decoder [24] allowing only for diversity of two but with a data rate of four. Gaining such insight, the following conjecture can be made.

Conjecture 1. Given a wireless communications system with $N_{T}$ transmit and $N_{R}$ receive antennas in a flat Rayleigh fading environment with maximum diversity $N_{R} N_{T}$ (see also [25] for definition), an Alamoutization scheme can be found with diversity order $D$ and data rate $R$, if $D \in \mathbb{N}$ and $R \in \mathbb{N}$ approximately factorizing the maximum diversity, that is, $D R \approx$ $N_{T} N_{R}$.

Note that this statement was not formulated in terms of a lemma since it may not be exactly true in the sense that exactly a diversity of say eight is obtained when actually only 6.4 is achieved. It is thus to apply with some care. On non-flatfading channels, the UMTS transmission allows the diversity to increase by assigning a number of fingers to each major energy contribution in the impulse response. In this case, all finger values are combined in a correspondingly larger matrix $\mathbf{H}$. However, $\mathbf{H}^{H} \mathbf{H}$ remains of the same size as before. The various fingers only contribute to higher diversity gain allowing to utilize BLAST schemes in which $\mathbf{H}^{H} \mathbf{H}$ would not be of full rank in a flat Rayleigh scenario.

\section{CONCLUSION}

In this paper, several extensions to the Alamouti space-time block code supporting very high transmit and receiver diversity have been proposed and their performance is evaluated. By combining conventional BLAST and new extended Alamouti schemes, a trade-off between diversity gain (and thus QoS) and supported data rate is offered allowing very high flexibility while the receiver complexity is kept approximately proportional to the transmitted data rate.

Not considered in this paper is the influence of the modulation scheme on the diversity. It is well known [8] that a rank criterion on the modulation scheme needs to be satisfied in order achieve the full diversity. In QPSK transmission, this rank criterion is, for example, not satisfied in the fourand eight-antenna transmission schemes. In other words, for some transmitted symbols, the full diversity will not be achieved. One can exclude such symbols or use different modulation schemes. In $[26,27]$, the possibility to use offset QPSK was proposed. This can be implemented in UMTS without sacrificing much of the existent hardware solutions. Another possibility very suitable for UMTS is to work with feedback schemes. In $[28,29]$, it is shown for the $4 \times 1$ as well as the $8 \times 1$ antenna scheme that a very simple feedback scheme retransmitting only one or two bits can reinstall the full diversity.

\section{APPENDICES}

\section{A. PROOFS FOR LEMMAS 2 AND 3}

Starting from the definition of $X$ in (19), it is observed that the squares in the denominator can be appended:

$$
X+1=\frac{\left|h_{1}+h_{4}\right|^{2}+\left|h_{2}-h_{3}\right|^{2}}{\left|h_{1}\right|^{2}+\left|h_{2}\right|^{2}+\left|h_{3}\right|^{2}+\left|h_{4}\right|^{2}} .
$$

In the case of i.i.d. complex-valued Gaussian distributed variables $h_{i}$, the two variates $h_{1}+h_{4}$ and $h_{2}+h_{3}$ become complex Gaussian distributed and independent of each other. They depend, however, on the variates in the nominator. Now, a linear orthogonal coordinate transformation is defined:

$$
\begin{array}{rlrl}
u & =\frac{\left(h_{1}+h_{4}\right)}{\sqrt{2}}, & v & =\frac{\left(h_{2}-h_{3}\right)}{\sqrt{2}}, \\
u^{\prime} & =\frac{\left(h_{1}-h_{4}\right)}{\sqrt{2}}, & v^{\prime}=\frac{\left(h_{2}+h_{3}\right)}{\sqrt{2}},
\end{array}
$$

such that $\sum_{i=1}^{4}\left|h_{i}\right|^{2}=|u|^{2}+|v|^{2}+\left|u^{\prime}\right|^{2}+\left|v^{\prime}\right|^{2}$ and

$$
\frac{X+1}{2}=\frac{|u|^{2}+|v|^{2}}{|u|^{2}+|v|^{2}+\left|u^{\prime}\right|^{2}+\left|v^{\prime}\right|^{2}}=\frac{X_{1}^{2}}{X_{1}^{2}+X_{2}^{2}} .
$$

Generally, if $X_{1}^{2}$ and $X_{2}^{2}$ are independent random variables following chi-square distributions with $\nu_{1}$ and $\nu_{2}$ degrees of freedom, respectively, then $X_{1}^{2} /\left(X_{1}^{2}+X_{2}^{2}\right)$ is said to follow a $\operatorname{beta}(p, q)$ distribution with $\nu_{1}=2 p$ and $\nu_{2}=2 q$ degrees of freedom and the probability density is given by $(1 / \mathrm{B}(p, q)) \xi^{p-1}(1-\xi)^{q-1}$, with $p=\nu_{1} / 2, q=\nu_{2} / 2$. This matches our case (A.3) for $\nu_{1}=\nu_{2}=4$ and the probability density specializes to $6 \xi(1-\xi)$. Transforming this back to $X$ gives the probability density

$$
f_{X}(x)= \begin{cases}\frac{3}{4}\left(1-x^{2}\right) & \text { for }|x|<1 \\ 0 & \text { elsewhere. }\end{cases}
$$

The independency of $X$ and $\eta=h^{2}$ can be established by transformation of variables starting from the two independent variates $Z_{i}=X_{i}^{2}$ defined above which are (up to a scaling) $\chi_{4}^{2}$ distributed, that is, their joint probability density is given by

$$
f_{Z_{1} Z_{2}}\left(z_{1}, z_{2}\right)=\frac{1}{16} z_{1} z_{2} e^{-\left(z_{1}+z_{2}\right) / 2} \text { for } z_{1}>0, z_{2}>0 .
$$

The $2 \times 2$ transformation between the variates $X, \eta$ and $Z_{1}$, $Z_{2}$ is derived from (A.3):

$$
\left\{\begin{array}{c}
X=\frac{Z_{1}-Z_{2}}{Z_{1}+Z_{2}} \\
\eta=Z_{1}+Z_{2}
\end{array}\right\}, \quad\left\{\begin{array}{l}
Z_{1}=\frac{1+X}{2} \eta \\
Z_{2}=\frac{1-X}{2} \eta
\end{array}\right\} .
$$

The rules for transformation of variates result in the follow- 
ing joint probability density for $X, \eta$ :

$$
\begin{aligned}
f_{X, \eta}(x, \eta) & =\frac{1}{64}\left(1-x^{2}\right) \eta^{3} e^{-\eta / 2} \\
& =f_{X}(x) f_{\eta}(\eta), \quad \text { for }|x|<1, \eta>0,
\end{aligned}
$$

where $f_{X}$ is given above and $f_{\eta}$ is the $\chi_{8}^{2}$-density rescaled to unit mean, that is,

$$
f_{\eta}(\eta)= \begin{cases}\frac{128}{3} \eta^{3} \mathrm{e}^{-4 \eta} & \text { for } \eta>0 \\ 0 & \text { elsewhere }\end{cases}
$$

For the $\mathrm{ZF}$ receiver (where $\mu=0$ ), it follows that the noise is increased by a factor of

$$
\mathrm{E}\left[\delta_{4}\right]=\mathrm{E}\left[\frac{1}{1-X^{2}}\right]=\int_{0}^{2} \lambda^{-1} f_{X}(\lambda-1) d \lambda=\frac{3}{2} .
$$

For the general linear receiver with $\mu>0$ (including the MMSE),

$$
\mathrm{E}\left[\delta_{4}\right]=\frac{1}{2} \mathrm{E}\left[\frac{1+X}{\left(1+\mu / h^{2}+X\right)^{2}}+\frac{1-X}{\left(1+\mu / h^{2}-X\right)^{2}}\right]
$$

is evaluated by exploiting independency of $X$ and $\eta=h^{2}$ :

$$
\mathrm{E}\left[\delta_{4}\right]=\int_{\eta=0}^{\infty} \int_{x=-1}^{1} \frac{1+x}{(1+\mu / \eta+x)^{2}} f_{X}(x) f_{\eta}(\eta) d x d \eta
$$

The integration over $x$ is straightforward. The remaining integral

$$
\begin{gathered}
\mathrm{E}\left[\delta_{4}\right]=32 \int_{0}^{\infty}\left(2 \eta^{2}+6 \mu \eta-\mu(3 \mu+4 \eta) \log (2 \eta+\mu)\right. \\
+\mu(3 \mu+4 \eta) \log \mu) \eta e^{-4 \eta} d \eta
\end{gathered}
$$

is evaluated in terms of the exponential integral which leads to $(32)$.

\section{B. SOME PROPERTIES OF HH ${ }^{H}$}

In the following it will be shown that all entries of $\mathbf{H}^{H} \mathbf{H}$ are real valued. The proof is performed by induction. Using block matrix notation $\mathbf{H}^{H} \mathbf{H}$ at a certain level $m$ equals

$$
\mathbf{H}^{H} \mathbf{H}=\left[\begin{array}{cc}
\mathbf{H}_{1}^{H} \mathbf{H}_{1}+\mathbf{H}_{2}^{H} \mathbf{H}_{2} & \mathbf{H}_{1}^{H} \mathbf{H}_{2}-\mathbf{H}_{2}^{T} \mathbf{H}_{1}^{*} \\
\mathbf{H}_{2}^{H} \mathbf{H}_{1}-\mathbf{H}_{1}^{T} \mathbf{H}_{2}^{*} & \mathbf{H}_{1}^{H} \mathbf{H}_{1}+\mathbf{H}_{2}^{H} \mathbf{H}_{2}
\end{array}\right]
$$

Thus, if the property is given at the lower level scheme, $\mathbf{H}_{1}^{H} \mathbf{H}_{1}$ and $\mathbf{H}_{2}^{H} \mathbf{H}_{2}$ are real valued and so are the diagonal block matrices. In the next step, the recursion for the nondiagonal block matrix $\mathbf{H}_{1}^{H} \mathbf{H}_{2}-\mathbf{H}_{2}^{T} \mathbf{H}_{1}^{*}$ is investigated. Assuming $\mathbf{H}_{1}$ is constructed by $\mathbf{H}_{11}$ and $\mathbf{H}_{12}$ and $\mathbf{H}_{2}$ in a similar manner by the matrices $\mathbf{H}_{21}$ and $\mathbf{H}_{22}$, then the term $\mathbf{H}_{1}^{H} \mathbf{H}_{2}$ is given by

$$
\mathbf{H}_{1}^{H} \mathbf{H}_{2}=\left[\begin{array}{ll}
\mathbf{H}_{11}^{H} \mathbf{H}_{21}+\mathbf{H}_{12}^{T} \mathbf{H}_{22}^{*} & \mathbf{H}_{11}^{H} \mathbf{H}_{22}-\mathbf{H}_{12}^{T} \mathbf{H}_{21}^{*} \\
\mathbf{H}_{12}^{H} \mathbf{H}_{21}-\mathbf{H}_{11}^{T} \mathbf{H}_{22}^{*} & \mathbf{H}_{12}^{H} \mathbf{H}_{22}+\mathbf{H}_{11}^{T} \mathbf{H}_{21}^{*}
\end{array}\right]
$$

and the nondiagonal block matrix is obtained by such value minus its transposed form $\mathbf{H}_{2}^{T} \mathbf{H}_{1}^{*}$. Thus, every term $\mathbf{H}_{1}^{H} \mathbf{H}_{2}-$ $\mathbf{H}_{2}^{T} \mathbf{H}_{1}^{*}$ is replaced by a sum of terms of the form $\mathbf{H}_{k l}^{H} \mathbf{H}_{m n}-$ $\mathbf{H}_{m n}^{T} \mathbf{H}_{k l}^{*}$. If the property holds for the level below, it also holds for the current level. To complete the induction argument, it has to be shown that the property also holds for the first level $(m=1)$. In this case, the diagonal elements are $\left|h_{1}\right|^{2}+\left|h_{2}\right|^{2}$ and the nondiagonal values are $h_{1}^{*} h_{2} \pm h_{2} h_{1}^{*}$, that is, either zero or $2 \mathfrak{R}\left\{h_{1}^{*} h_{2}\right\}$. Thus, all entries are real valued. Note that due to the different signs occurring, it cannot be concluded that the terms become zero.

The second property is shown in [30]. For the third property, it is observed that every nondiagonal term $X$ consists of either elements $\left(h_{i} h_{k}^{*}+h_{i}^{*} h_{k}\right) / h^{2}$ or $-\left(h_{l} h_{m}^{*}+\right.$ $\left.h_{l}^{*} h_{m}\right) / h^{2}$. Thus building $X+1$ allows to consider $\left(h^{2}+h_{i} h_{k}^{*}+\right.$ $\left.h_{i}^{*} h_{k}\right) / h^{2}$ and $\left(h^{2}-h_{l} h_{m}^{*}+h_{l}^{*} h_{m}\right) / h^{2}$, further allowing to reorganize the terms into $\left(h_{i}+h_{k}\right)\left(h_{i}+h_{k}\right)^{*} / h^{2}$ and $\left(h_{l}+\right.$ $\left.h_{m}\right)\left(h_{l}-h_{m}\right)^{*} / h^{2}$. By applying the same transformation as in Appendix A:

$$
\begin{aligned}
u=\frac{\left(h_{i}+h_{k}\right)}{\sqrt{2}}, & v=\frac{\left(h_{l}-h_{m}\right)}{\sqrt{2}}, \\
u^{\prime}=\frac{\left(h_{i}-h_{k}\right)}{\sqrt{2}}, & v^{\prime}=\frac{\left(h_{l}+h_{m}\right)}{\sqrt{2}},
\end{aligned}
$$

the terms $X+1$ can be written in terms of independent Gaussian variables and the same rules as before apply. The resulting term then reads $(X+1) / 2=X_{1}^{2} /\left(X_{1}^{2}+X_{2}^{2}\right)$ with $X_{1}$ and $X_{2}$ being $\chi^{2}$-distributed with $v=2^{m}=N_{T}$ degrees of freedom each and

$$
\frac{1}{\mathrm{~B}\left(N_{T} / 2, N_{T} / 2\right)} \xi^{N_{T} / 2-1}(1-\xi)^{N_{T} / 2-1}
$$

is obtained, resulting in the density (49) for $X$.

\section{ACKNOWLEDGMENTS}

The authors like to thank Maja Lončar, Lund University of Technology, Ralf Müller, ftw., as well as Gerhard Gritsch, Vienna University of Technology, for their helpful comments. This work was carried out with funding from $\mathbf{K}$ plus in the ftw. project C3 "Smart Antennas for UMTS Frequency Division Duplex" together with Infineon Technologies and Austrian Research Centers, Seibersdorf (ARCS).

\section{REFERENCES}

[1] Third Generation Partnership Project, "Transmitter diversity solutions for multiple antennas, version 1.0.2," 3GPP Technical Report 25.869, Technical Specification Group Radio Access Network, March 2002.

[2] Third Generation Partnership Project, "Multiple Input Multiple Output (mimo) antennae processing for HSDPA, version 1.1.0," Tech. Rep. 25.876, Technical Specification Group Radio Access Network, February 2002. 
[3] A. F. Naguib, N. Seshadri, and A. R. Calderbank, "Increasing data rate over wireless channels," IEEE Signal Processing Magazine, vol. 17, no. 3, pp. 76-92, 2000.

[4] T. H. Liew and L. Hanzo, "Space-time codes and concatenated channel codes for wireless communications," Proceedings of the IEEE, vol. 90, no. 2, pp. 187-219, 2002.

[5] T. Matsumoto, J. Jlitaro, and M. Juntti, "Overview and recent challenges towards multiple-input multiple-output communications systems," IEEE Vehicular Technology Society News, vol. 50, no. 2, pp. 4-9, 2003.

[6] G. J. Foschini and M. J. Gans, "On limits of wireless communication in a fading environment when using multiple antennas," Wireless Personal Communications, vol. 6, no. 3, pp. 311-335, 1998

[7] I. E. Telatar, "Capacity of multi-antenna Gaussian channels," European Transactions on Telecommunications, vol. 10, no. 6, pp. 585-595, 1999, Technical Memorandum, Bell Laboratories, Lucent Technologies, October 1998.

[8] V. Tarokh, N. Seshadri, and A. R. Calderbank, "Space-time codes for high data rate wireless communication: performance criterion and code construction," IEEE Transactions on Information Theory, vol. 44, no. 2, pp. 744-765, 1998.

[9] B. Hassibi and B. M. Hochwald, "Linear dispersion codes," in Proc. IEEE International Symposium on Information Theory, $\mathrm{p}$. 325, Wash, DC, USA, 2001.

[10] B. Hassibi and B. M. Hochwald, "High-rate codes that are linear in space and time," IEEE Transactions on Information Theory, vol. 48, no. 7, pp. 1804-1824, 2002.

[11] S. M. Alamouti, "A simple transmit diversity technique for wireless communications," IEEE Journal on Selected Areas in Communications, vol. 16, no. 8, pp. 1451-1458, 1998.

[12] B. Hochwald, T. L. Marzetta, and C. B. Papadias, "A transmitter diversity scheme for wideband CDMA systems based on space-time spreading," IEEE Journal on Selected Areas in Communications, vol. 19, no. 1, pp. 48-60, 2001.

[13] J. G. Proakis, Digital Communications, McGraw-Hill, New York, NY, USA, 4th edition, 2001.

[14] J. H. Winters, J. Salz, and R. D. Gitlin, "The impact of antenna diversity on the capacity of wireless communication systems," IEEE Trans. Communications, vol. 42, no. 2-4, pp. 1740-1751, 1994.

[15] M. Rupp, C. F. Mecklenbräuker, and G. Gritsch, "High diversity with simple space time block-codes and linear receivers," in Proc. IEEE Global Telecommunications Conference, pp. 302 306, San Francisco, Calif, USA, December 2003.

[16] G. Gritsch, H. Weinrichter, and M. Rupp, "Understanding the BER performance of space-time block codes," in Proc. IEEE Signal Processing Advances in Wireless Communications, pp. 400-404, Rome, Italy, June 2003.

[17] C. C. Martin, J. H. Winters, and N. R. Sollenberger, "MIMO radio channel measurements: performance comparison of antenna configurations," in Proc. IEEE 54th Vehicular Technology Conference, vol. 2, pp. 1225-1229, Atlantic City, NJ, USA, October 2001.

[18] M. Rupp and C. F. Mecklenbräuker, "Improving transmission by MIMO channel structuring," in Proc. IEEE International Conference on Communications, vol. 5, pp. 3066-3070, Anchorage, Alaska, USA, May 2003.

[19] C. B. Papadias and G. J. Foschini, "A space-time coding approach for systems employing four transmit antennas," in Proc. IEEE Int. Conf. Acoustics, Speech, Signal Processing, vol. 4, pp. 2481-2484, Salt Lake City, Utah, USA, 2001.

[20] I. S. Gradsteyn and I. M. Ryzhik, Table of Integrals, Series and Products, Academic Press, New York, NY, USA, 1980.

[21] P. W. Wolniansky, G. J. Foschini, G. D. Golden, and R. A. Valenzuela, "V-BLAST: an architecture for realizing very high data rates over the rich-scattering wireless channel," in Proc. International Symposium Signals, Systems, and Electronics, pp. 295-300, Pisa, Italy, September 1998.

[22] G. Gritsch and H. Weinrichter, "Adaptive subspace modulation in spatially correlated MIMO channels," in Proc. IEEE 13th International Symposium on Personal, Indoor, and Mobile Radio Communications, vol. 4, pp. 1772-1776, Lisboa, Portugal, September 2002.

[23] G. Gritsch, H. Weinrichter, and M. Rupp, "Two adaptive coded MIMO systems exploiting partial channel knowledge at the transmitter," in Proc. of SSC 04, pp. 31-38, Erlangen, Germany, January 2004.

[24] R. van Nee, A. van Zelst, and G. Awater, "Maximum likelihood decoding in a space division multiplexing system," in Proc. IEEE 51st Vehicular Technology Conference, vol. 1, pp. 610, Tokyo, Japan, May 2000.

[25] J. B. Andersen, "Array gain and capacity for known random channels with multiple element arrays at both ends," IEEE Journal on Selected Areas in Communications, vol. 18, no. 11, pp. 2172-2178, 2000.

[26] M. Rupp and C. F. Mecklenbräuker, "On extended Alamouti schemes for space-time coding," in Proc. 5th International Symposium on Wireless Personal Multimedia Communications, pp. 115-119, Honolulu, Hawaii, USA, October 2002.

[27] M. M. da Silva and A. Correia, "Space time block coding for 4 antennas with coding rate 1," in Proc. IEEE 17th International Symposium on Spread Spectrum Techniques and Applications, pp. 318-322, Prague, Czech Republic, September 2002.

[28] B. Badic, M. Rupp, and H. Weinrichter, "Quasi-orthogonal space-time block codes for data transmission over four and eight transmit antennas with very low feedback rate," in Proc. of SCC 04, pp. 157-164, Erlangen, Germany, January 2004.

[29] B. Badic, M. Rupp, and H. Weinrichter, "Adaptive channel matched extended alamouti space-time code exploiting partial feedback," in 8th Conference on CIC, p. 350, Seoul, October 2003.

[30] A. Stamoulis, Z. Liu, and G. B. Giannakis, "Space-time blockcoded OFDMA with linear precoding for multirate services," IEEE Trans. Signal Processing, vol. 50, no. 1, pp. 119-129, 2002.

Christoph F. Mecklenbräuker was born in Darmstadt, Germany, in 1967. He received his Dipl.Ing. degree in electrical engineering from Vienna University of Technology in 1992 and the Dr.Ing. degree from Ruhr University of Bochum in 1998, respectively. His doctoral thesis on matched field processing was awarded with the Gert Massenberg Prize. He worked for the Mobile Networks Radio Department of Siemens AG, where he participated in the European framework of ACTS 90 "Frames." He was a delegate to the Third Generation Partnership Project (3GPP) and engaged in the standardization of the radio access network for UMTS. Since 2000, he holds a Senior Research position at the Telecommunications Research Center Vienna (ftw.) in the field of mobile communications. Currently, he gives a course at the Vienna Technical University on $3 \mathrm{G}$ mobile networks. He has authored around 60 papers in international journals and conferences, for which he has also served as a reviewer and holds 8 patents in the field of mobile cellular networks. His current research interests include antenna array and MIMO signal processing for mobile communications. 
Markus Rupp received his Diploma degree in electrical engineering from FHS Saarbrücken, Germany, and the University of Saarbrücken in 1984 and 1988, respectively, and the Doctoral degree (summa cum laude) in acoustical echo compensation from the Technische Universität (TH) Darmstadt, Germany, in 1993. He was awarded a DAAD postdoctoral fellowship and spent the time from November 1993

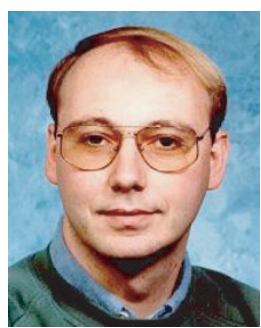
until September 1995 in the Department of Electrical and Computer Engineering, University of California, Santa Barbara, working on a robustness theory for adaptive filters. From October 1995 until August 2001, he has been with Bell Labs, Lucent Technologies (before AT\&T), Wireless Research Lab, Holmdel, NJ, working on wireless phones and implementation issues of wireless modems. In May 1999, he moved to The Netherlands, joining Bell Labs efforts in wireless LANs in Europe. In October 2001, he joined the Faculty of Electrical Engineering and Information Technology as a Full Professor at the TU Wien, Vienna, Austria. He is currently involved in rapid prototyping methods for wireless systems as well as MIMO coding and equalization techniques. He has more than 100 publications and patents in the fields of adaptive filters and wireless systems. 